\title{
Water and biological time keeping
}

\author{
D. J. Morré \& D. M. Morré \\ NOX Technologies, Inc. and Purdue University, West Lafayette, USA
}

\begin{abstract}
A homodimeric, growth-related and time-keeping hydroquinone oxidase (ENOX1) of the eukaryotic cell surface capable of oxidizing extracellular NADH exhibits properties of the ultradian driver of the biological $24 \mathrm{~h}$ circadian clock by exhibiting a complex $2+3$ set of oscillations with a period length of $24 \mathrm{~min}$. The oscillations require bound copper, are recapitulated by aqueous solutions of copper salts and appear to derive from 30 to $40 \mathrm{sec}$ periodic variations in the ratios of ortho and para nuclear spins of the paired hydrogen atoms of the elongated octahedral structure of the ENOX2 protein bound copper ${ }^{\text {II }}$ hexahydrates. Based on a heartbeat model of limit cycle oscillations, these oscillations correlate with generation of the 24 min periodicity as a form of carrier wave. A corollary of these observations is that the ortho-para oscillations must occur in a highly synchronized manner. Attendant oscillatory changes in redox potential offer an opportunity to monitor oscillations in synchronous populations of water molecules. The periodicity of the ENOX1/copper/water clock can be phased by brief 10 to $20 \mathrm{sec}$ exposure to very low frequency electromagnetic fields. The synchronized populations of oscillating water molecules give rise to oscillatory electromagnetic fields that apparently are perceived by adjacent water molecules to create a collectively coherent synchronous system. Two asynchronous water samples placed adjacent to one another but separated by a thin non-metal barrier become fully synchronized in a matter of several min. A barrier of metal foil prevents the synchronization. The corollary of these observations is that contiguous water molecules function synchronously perhaps even over relatively long distances. Two samples of water from contiguous still or flowing bodies of water collected from different locations and analyzed simultaneously were synchronous in their oscillations in redox potential measured as changes in rates of NADH oxidation. Our findings suggest that water molecules communicate with each other via very low frequency electromagnetic fields. These fields appear to be generated by the
\end{abstract}


energetics of synchronous ortho-para interconversions of the nuclear spin pairs of the paired water hydrogens. The level of synchrony achieved may translate into highly coordinated populations of coherent water perhaps more extensive than previously anticipated.

Keywords: coherent water, biological time keeping, low frequency electromagnetic fields, oscillations in ortho-para nuclear spins of paired hydrogen atoms of water, limit oscillators.

\section{Introduction}

Our laboratory discovered and described an ultradian oscillator of the cell's biological clock with a first paper in 1998 [1] and a major paper in 2002 [2]. Since then we have focused on the mechanism underlying the oscillations which require the association of two copper hexahydrates per monomer of the oscillator (ENOX1) protein with NADH oxidase and protein disulfide thiol-interchange activities. The functional unit is a dimer located on the cell surface that is also shed into the circulation. The period length of $24 \mathrm{~min}$ is independent of temperature and repeats 60 times per day. Changes in period length to 22, 36 or $42 \mathrm{~min}$ as a result of site directed mutagenesis of recombinant ENOX proteins result in circadian days based on measurements of GADPH of 22, 36 or $42 \mathrm{~h}$ in transfected cells. The period length is increased to $30 \mathrm{~min}$ in heavy water as is characteristic of clock-related phenomena. The protein has been cloned [3]. The period length of the protein oscillator is phased by light and by melatonin. The 24 min oscillations are given by copper ${ }^{\text {II }}$ in solution. The potential energy change is sufficient to drive NADH oxidation. Oscillations are physical rather than chemical to explain the temperature independence of period length and subsequently traced to a metastable equilibrium condition in the ratio of ortho to para nuclear spin orientation of the water-associated hydrogen atoms of the copper hexahydrate. Copper ${ }^{\mathrm{II}}$ oscillations are unresponsive to light and melatonin but must somehow also be phased. Phasing of the ENOX1/copper clock is accomplished by brief exposure to very low frequency EMF [4].

\section{Methods}

To determine phase shifts due to low frequency EMF, measurements of redox potential or redox potential-catalyzed oxidation of NAD were in parallel on two aliquots of the same preparation, one exposed to EMF and the other not. Analyses were in parallel using duplicate Hitachi U3210 spectrophotometers such that EMF exposure was the only variable.

As a test for water synchrony, oscillatory changes in redox potential, as evidenced by corresponding oscillations in rates of NADH oxidation, were monitored. For example, $120 \mu \mathrm{M}$ NADH prepared in HPLC grade water was placed in two plastic spectrometer cuvettes. One of the cuvettes was exposed to low frequency EMF $(30 \mathrm{sec}, 40 \mu \mathrm{T})$ to be out of phase with the water in the other cuvette. When placed side by side for several min, subsequent measurements revealed identical oscillatory patterns for the water in the two cuvettes when 
analyzed in parallel using the two paired spectrophotomers. The findings are indicative of a property of out-of-phase water samples to communicate via EMF to achieve phase synchrony in a matter of minutes.

\section{Results}

A natural oscillatory cycle inherent to liquid water is proposed as the basis for the biological clock, the evidence for which has evolved experimentally from the clock-related protein-associated copper hexahydrate [3]. The likely candidate resides in instabilities in the water molecule itself, whose two hydrogen nuclei undergo ordered periodic shifts between ortho and para states [5]. However, until now, there has been no clear hypothesis as to how the ratio of ortho to para water departs from equilibrium and is maintained in a regular pattern of oscillations. As para water forms, it might locally add to the field generated by the collective order which would further favor the accumulation of para water for some period of time. But then perhaps the initial conditions favoring para water formation as a result of this process produces an increased potential which cannot be released or discharged until it achieves some minimum threshold of energy of activation. Once the minimum threshold is achieved, there is a discharge of potential and the conditions favorable to formation of ortho water result. The process now runs for a time in the opposite direction until some threshold level of ortho water is achieved and the cycle reverses. These are characteristics of limit cycle oscillators that exhibit large deviations from equilibrium. In a limit cycle oscillator, the amplitude tends to be more or less constant but the frequency may vary. Control of heart beat is the classic example of a limit cycle oscillator. Figure 1 shows a diagram to explain ortho-para oscillations in water by analogy to the limit cycle oscillatory control of heart beat rate.

The key that makes the rhythmic firing of pacemaker cells of the heart an attractive model of ortho-para water oscillations is that, unlike other muscles cells and neurons, the pacemaker cells slowly depolarize by themselves. Depolarization, a reduction in the degree of electronegativity in a resting cell (the cells become more positive) occurs when positively charged sodium and calcium ions enter the cytosol to generate inward ionic currents. Depolarization continues until the threshold potential is reached between $-40 \mathrm{mV}$ and $-50 \mathrm{mV}$. When the threshold is reached, the cells enter phase 0 or upstroke. Following upstroke, the cardiomyocytes repolarize. Repolarization, the return to resting potential, occurs when outward currents restore the membrane potential to its resting level of electronegativity. In the heart, repolarizing currents are generated largely by potassium efflux, possibly supplemented by the inward movement of chloride ions. The only atomic phenomenon that occurs on a similar time scale is the equilibration of the two alternative orientations of the nuclear spins (ortho and para) of the hydrogen atoms of water [6].

Spectral evidence for ortho-para spin isomer disequilibrium in liquid water has been provided by Pershin [7, 8] (fig. 2). The spectrometric measurements were of backscattered Raman signal of water excited by the second-harmonic 
Highest energy threshold

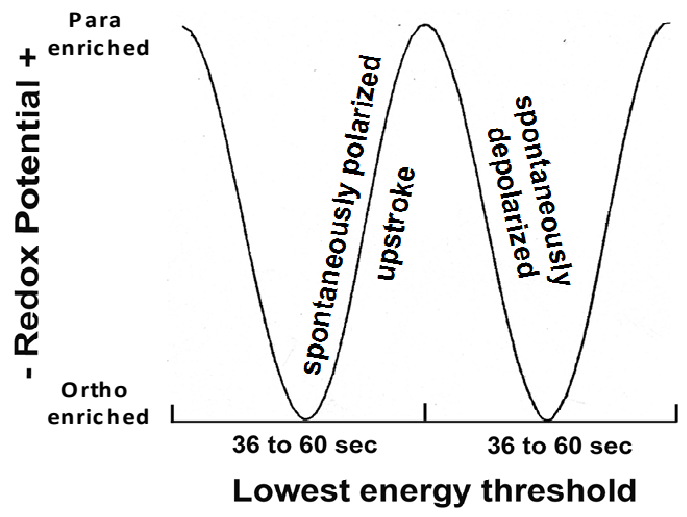

Figure 1: Model based on the concept of limit oscillations to explain ortho-para oscillations and their failure to reach a steady-state equilibrium. Para water is suggested to reach some high energy threshold and then spontaneously converts to ortho water to reach some lower energy threshold at which time the conversion reverses back to para water.
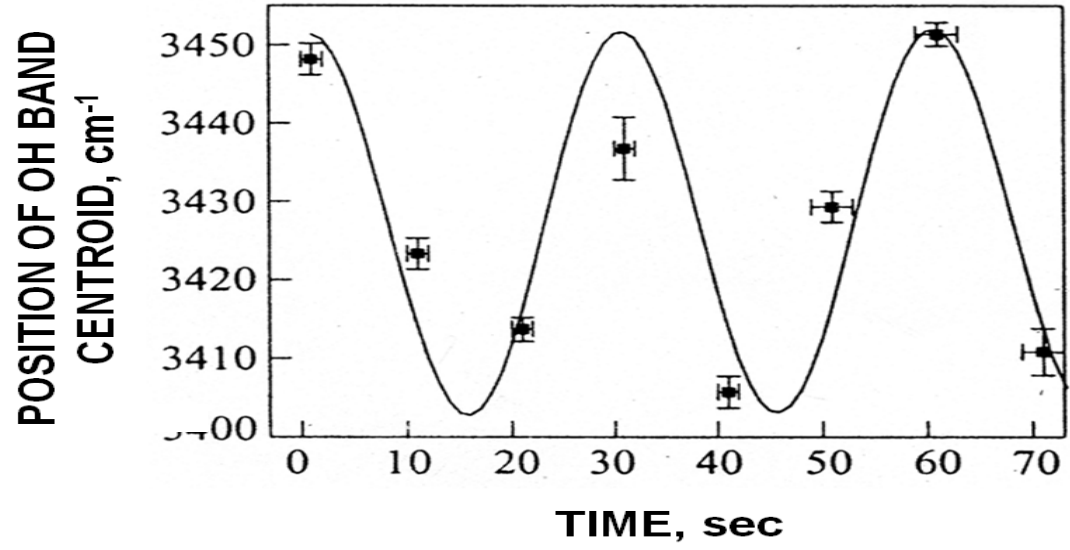

Figure 2: Harmonic oscillations of the $\mathrm{OH}$ centroid band determined from Raman scattering as a measure of exchange between two water states related to ortho/para ratios. The period length was determined to be $35 \pm 13 \mathrm{sec}$. From Pershin [7]. Reprinted with permission. 
radiation of a Nd:YAG laser (a single 10-ns pulse or a train of such pulses with a repetition period of one second). The laser radiation is focused in a cell with room-temperature water. The measurements are performed at laser intensities of $2,15,35$ and $350 \mathrm{MW} / \mathrm{cm}^{2}$. The Raman signal was focused on the entrance slit of a polychromator interfaced with an image intensifier and a diode array. Lowintensity measurements were carried out with a cooled diode array.

Spectral manifestation of a relatively high mobility of "hot" molecules were observed to result in the formation of a high-frequency wing of the $\mathrm{OH}$ band and a shift of the center of the band with a coefficient of about $1 \mathrm{~cm}^{-1} \operatorname{grad}^{-1}$ upon heating. Such a shift was interpreted as a decrease in the concentration of strong $\mathrm{H}$-bonds as a result of disordering through destruction of polymer water and an increase in the number of complexes whose molecules can rotate [7].

The result was the experimental validation of the hypothesis of independent existence of two liquids in water. Both liquids consist of hydrogen-bonded complexes of $\mathrm{H}_{2} \mathrm{O}$ molecules. Each exhibit characteristic frequency distributions of $\mathrm{OH}$ oscillators and form an integrated envelope of the $\mathrm{OH}$ stretching vibration band.

Evidence that water vapor is a mixture of two independent states of liquid water with long lifetimes and two hydrogen bond types differing in energy led Pershin to consider the two water states as independent fractions represented by ortho and para water respectively [7, personal communication]. Specifically "the energy of the hydrogen bond between ortho isomers of molecules which always rotate seems to be lower than between para molecules, a part of which cannot rotate at room temperature."

The conclusion was reached that this overheating-overcooling process may be a fundamental property of water, which manifests itself at any temperature and is not the result of perturbation of overcooled water by an optical pulse. Pershin's findings show "that such an evolution of the band center is steadily observed in water and at room temperature. Moreover, this overheating-overcooling process is approximated by a harmonic function with a period of $35 \pm 13 \mathrm{~s}$ without noticeable damping for $\sim 16$ min" (fig. 2 ).

The differences in energies of the different spin isomers of water are small (less than $10^{-24} \mathrm{erg}$ ) [9] and are much lower than the energy of thermal motion. Therefore, a spin-only interaction would not be expected to affect intermolecular interactions [9]. On the other hand, the absorption rates of ortho and para water from water vapor to various organic and inorganic sorbents have been observed to differ markedly. The binding of the para isomer with such preparations is distinctly faster than the binding of the ortho isomer. Binding avidity is most likely determined from differences in quantum statistics for the two different spin isomers as noted by Potekhin and Khusainova [10]. Estimates of the energy barriers that determine rates of absorption by these authors suggested that the difference in free energy barriers may exceed the energy of spin-spin and spinorbit interaction by many orders [10]. This raises the possibility that the spin state of water may substantially influence physical, chemical and biological phenomena including redox potential. 
A defining characteristic of the clock-related oscillations is a characteristic 2 +3 pattern of oscillations. Within each repeat of 5 oscillations, two are more widely spaced than the remaining three. Since the primary oscillations on average have a period length of only $30-50 \mathrm{~s}$, the $2+3$ pattern must represent a carrier wave generated by interaction of one to several parallel sets of primary oscillations of much greater frequency such as the ortho/para water oscillations with period lengths of 30 or $50 \mathrm{~s}$ reported by Pershin [7].

An alternative source of energy absorbed and emitted by water and correlated with ortho-para oscillation of ortho-para spin pairs on water auto-oscillations in luminescence following infrared radiation [11, fig. 3]. The emissions oscillate with period lengths of 300 and $1150 \mathrm{~s}$ that agree with our previously found period of oscillation for water of 18 min reflective of ortho to para spin isomers based on measurements of redox potential of pure water [5] (see also fig. 4).

Within the water molecule, nuclear spins of protons of water were considered as a primary target for external magnetic fields [12]. Proton spins were suggested

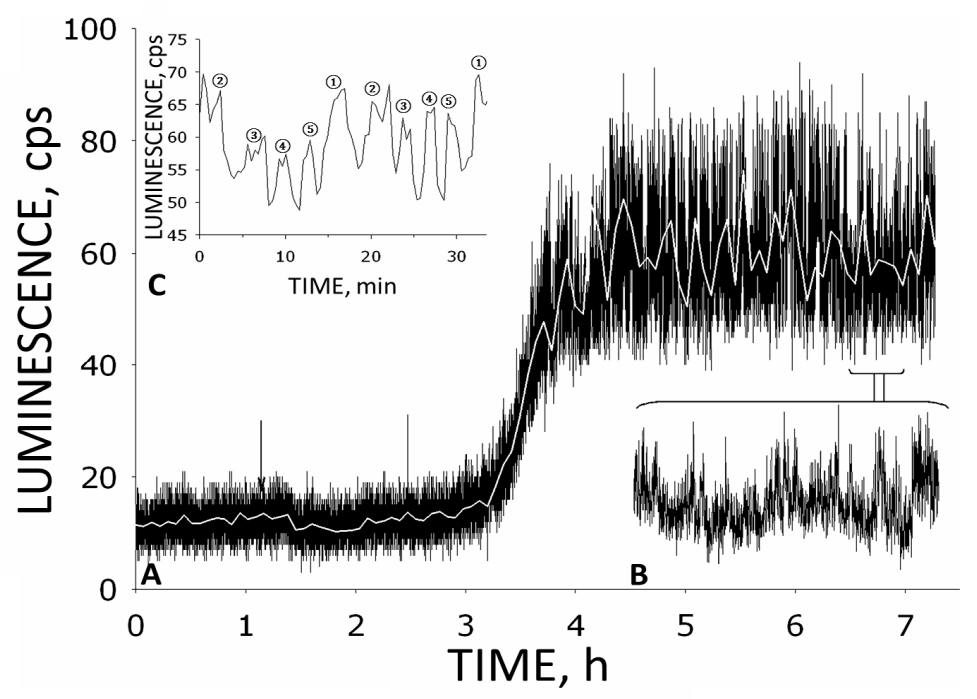

Figure 3: Effect of laser radiation on water luminescence. A, Data of a representative experiment. Water was exposed to an infrared laser $(\mathrm{A}=1264 \mathrm{~nm}$, power $5 \mathrm{~mW}$ ) for $5 \mathrm{~min}$. The time of exposure is shown by a vertical arrow. To the left of the arrow is a record of the background water luminescence before the onset of laser irradiation. The white line on the basic plot presents the macrostructure of a signal obtained using the smoothing option of the program SigmaPlot. B. The microstructure of changes in water luminescence. C. The integral intensity of water luminescence corresponding to that bracketed in B. Maxima labeled (1) to (5) denote an 18 min period length. (Modified from Gudkov et al. [11]. Reprinted with permission.) 


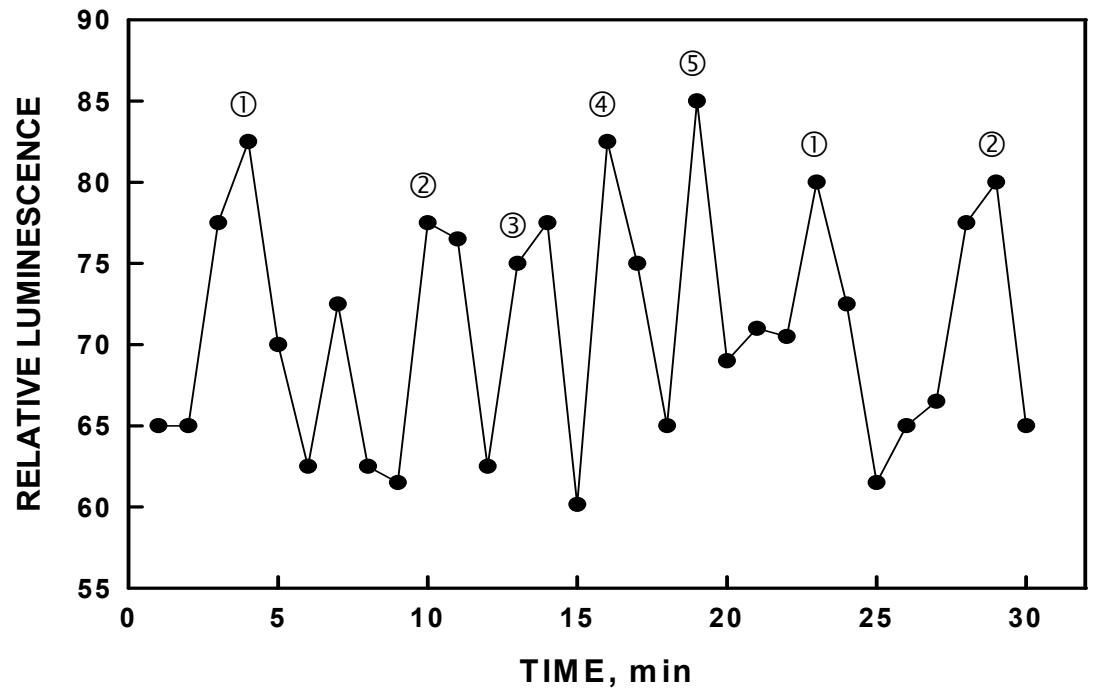

Figure 4: Relative luminescence measured independently but closely approximating the conditions of fig. 3 . The characteristic $2+3$ pattern of water redox oscillations (fig. 5) is recapitulated as in fig. 3C with a period length of $18 \mathrm{~min}$ in which maxima labeled (1) and (2) are separated by $6 \mathrm{~min}$.

to take part in spin-orbit interactions that would modify proton motion and influence the rate of formation and breakup of water clusters. Morré et al. [4] provide results from $\mathrm{Cu}^{\mathrm{II}} \mathrm{Cl}_{2}$ solutions to demonstrate that nuclear spin-dependent oscillations of water are phased by exposure to low frequency electromagnetic fields. Phasing by electromagnetic fields and use of rates of NADH oxidation have been combined to provide evidence for long range synchrony and communication among contiguous water molecules extending to bodies of water separated by considerable distances (figs. 5-7).

Irrespective of the mechanism, the ortho-para oscillations must occur in a highly synchronized manner. Moreover, attendant oscillatory changes in redox potential offer an opportunity to monitor, as well, synchrony of oscillations in populations of water molecules. The periodicity of the ENOX1/copper ${ }^{\mathrm{II}} /$ water clock can be phased by a brief 10 to $20 \mathrm{~s}$ exposure to very low frequency electromagnetic fields. In so doing, the synchronized populations of oscillating water molecules create a collectively coherent synchronous system. Two synchronous water samples separated by a non-metal barrier become fully synchronized within times even as short as 1 min such that water molecules, being dipoles with strong polar interactions and a high degree of spatial orientation, may not only respond to low frequency electromagnetic fields but may also generate such fields so that water molecules are able to communicate via the oscillating electromagnetic field and, therefore, remain highly synchronous perhaps over relatively long distances. 

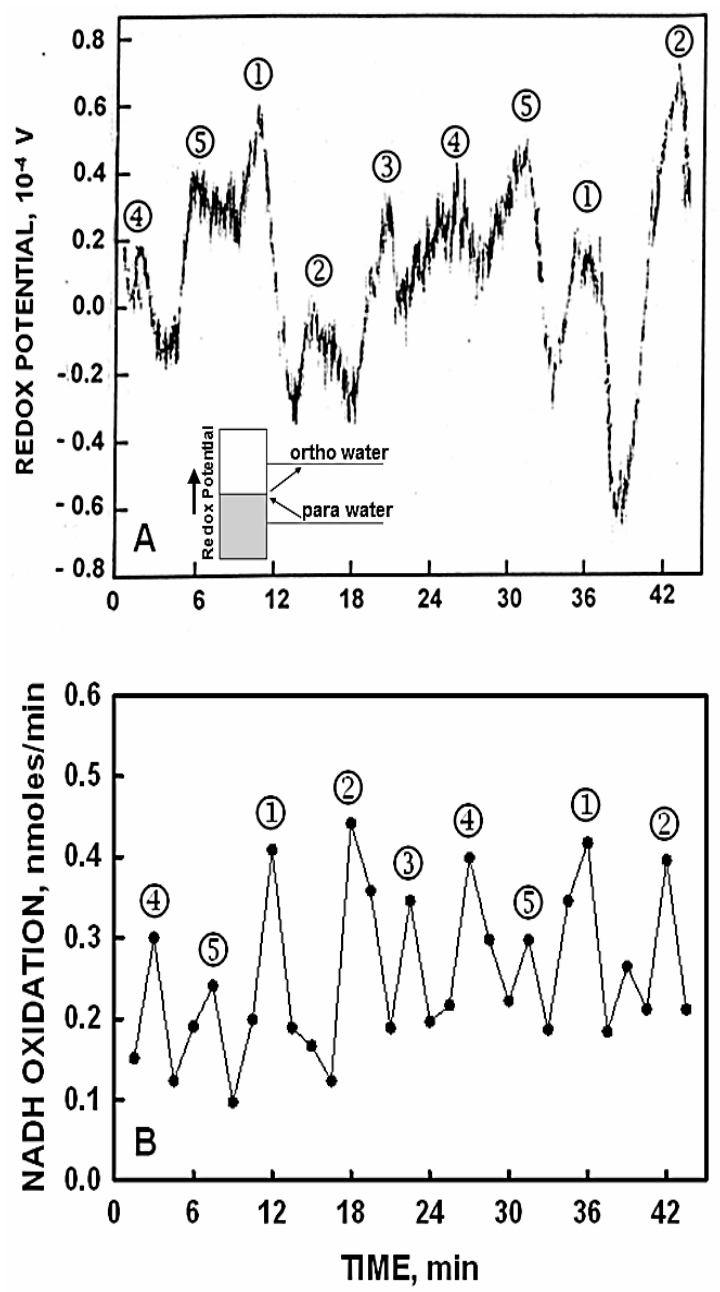

Figure 5: The redox potential of an aqueous solution of copper $^{\mathrm{II}}$ chloride measured continuously showing a $2+3$ pattern of oscillations with NADH oxidation measured over $1 \mathrm{~min}$ at intervals of $1.5 \mathrm{~min}$ in parallel. The period length of both is $24 \mathrm{~min}$. Results with pure water are similar except that the period length with pure water is 18 min. NADH oxidation was used to monitor oscillations in the redox potential of contiguous bodies of water to demonstrate water coherence over long distances (see text). 


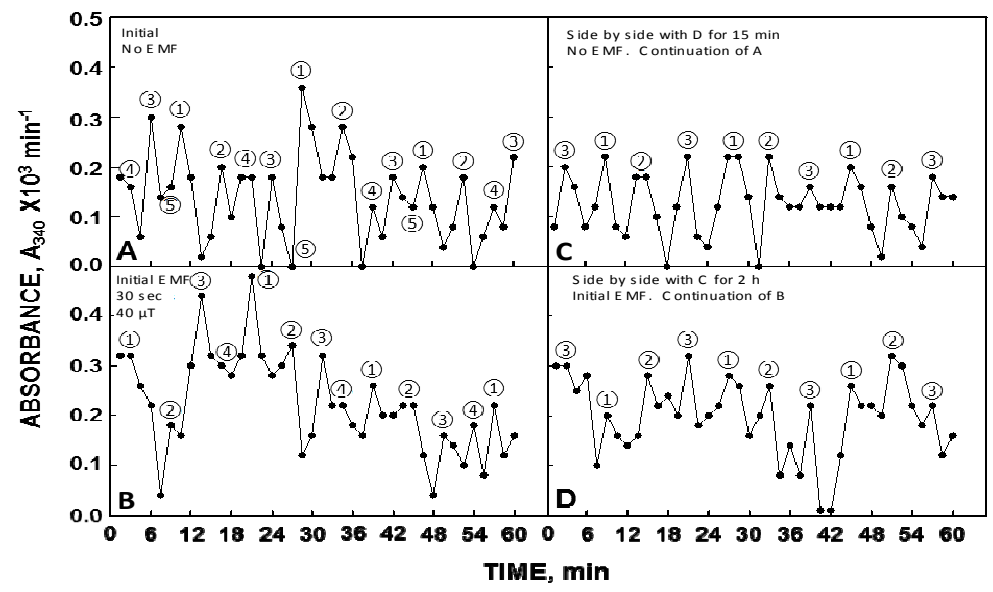

Figure 6: Low frequency EMF synchronization of asynchronous water samples through a thin plastic barrier. A. HPLC grade water. No EMF. B. A sample of HPLC grade water identical to that in A was phased using low frequency EMF $(30 \sec 40 \mu \mathrm{T})$ and shown to be out of phase with the original water sample (A). C, D. When the two plastic cuvettes containing the samples were placed adjacent to each other for $15 \mathrm{~min}$, both samples then began to oscillate synchronously.

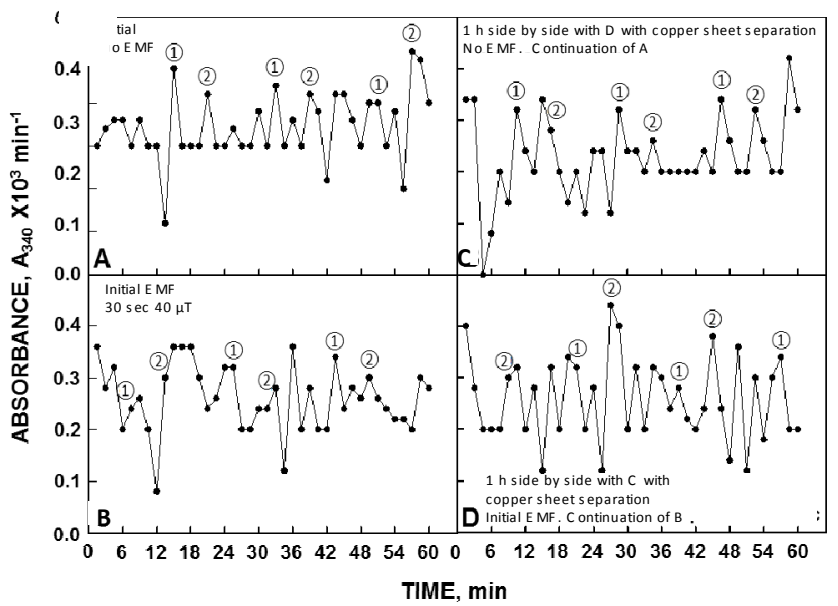

Figure 7: As in fig. 6 except that the two samples when placed side by side were separated by a thin barrier of sheet copper which blocked the low frequency EMF generated from the two adjacent water samples and prevented the phasing observed in fig. 6 for up to $2 \mathrm{~h}$. 
As a test for water synchrony, oscillatory changes in redox potential, as evidenced by corresponding oscillations in rates of NADH oxidation, were monitored (fig. 5). For example, $120 \mu \mathrm{M}$ NADH prepared in HPLC grade water was placed in two plastic spectrometer cuvettes. One of the cuvettes was exposed to low frequency electromagnetic field $(30 \mathrm{~s}, 40 \mu \mathrm{T})$ to be out of phase with the water in the other cuvette. When placed side by side for $15 \mathrm{~min}$, subsequent measurements revealed identical oscillatory patterns for the two cuvettes when analyzed in parallel using two paired spectrophotometers (fig. 6). When a thin copper or aluminum foil sheet was placed between the cuvettes for as long as $2 \mathrm{~h}$, the communication between the two cuvettes was blocked (fig. 7). The shortest exposure time sufficient to initiate full synchrony thus far tested was $5 \mathrm{~min}$.

Water sampled from two points in a shallow pond with sampling points separated by 100 feet $(6 \mathrm{~m})$ also were synchronous as was water collected from opposite ends of a 20 acre lake. Flowing water also was synchronous. Thus far the largest bodies of water sampled have been from Lake Ontario and from the Niagara River in New York with sampling points separated by approximately 20 miles for each [12]. Samples from both were found to be synchronous. There have been previous reports supporting the concept of coherent water $[13,14]$. Our findings support the concept that water coherence translates into highly correlated water populations but to an extent much greater than that which may have been previously anticipated.

\section{References}

[1] Morré, D.J. \& Morré, D.M. NADH oxidase activity of soybean plasma membranes oscillates with a temperature compensated period of $24 \mathrm{~min}$. Plant Journal 16, pp. 277-284, 1998.

[2] Morré, D.J, Chueh, P.J., Pletcher, J., Tang, X., Wu, L.Y. \& Morré, D.M., Biochemical basis for the biological clock. Biochemistry. 40, pp. 11941$11954,2002$.

[3] Jiang, Z., Gorenstein, N.M., Morré, D.M. \& Morré, D.J., Molecular cloning and characterization of a candidate human growth-related and timekeeping constitutive cell surface hydroquinone (NADH) oxidase. Biochemistry, 47, pp. 14028-14038, 2008.

[4] Morré, D.J., Jiang, Z., Marjanovic, M., Orczyk, J. \& Morré, D.M., Response of the regulatory oscillatory behavior of copper ${ }^{\mathrm{II}}$-containing ECTO-NOX proteins and of $\mathrm{Cu}^{\mathrm{II}} \mathrm{Cl}_{2}$ in solution to electromagnetic fields. Journal of Inorganic Biochemistry, 102, pp. 1812-1818, 2008.

[5] Morré, D.J., Orczyk, J., Hignite, H. \& Kim, C., Regular oscillatory behavior of aqueous solutions of $\mathrm{Cu}^{\mathrm{II}}$ salts related to effects on equilibrium dynamics of ortho/para hydrogen spin isomers of water. Journal of Inorganic Biochemistry, 102, pp. 260-267, 2008.

[6] Tikhonov, V.I. \& Volkov, A.A., Separation of water into its ortho and para isomers. Science, 296, p. 2363, 2002. 
[7] Pershin, S.M., Two-liquid water. Physics of Wave Phenomena, 13, pp. 192-208, 2005.

[8] Pershin, S.M. Harmonic oscillations of the concentration of H-bonds in liquid water. Laser Physics 16, pp. 114-119, 2006.

[9] Emsley, J.M., Feeney, J. \& Sutcliffe, L.H. High Resolution Nuclear Magnetic Resonance Spectroscopy, Vol. 1, Pergamon Press, Oxford, 1965.

[10] Potekhin, S.A. \& Khusainova, R.S., Spin-dependent absorption of water molecules. Biophysical Chemistry, 118, pp. 84-87, 2005.

[11] Gudkov, S.V., Brushov, V.I., Astrshev, M.E., Chernikov, A.V., Yaguzhinsky, L.S. and Zakharov, S.D., Oxygen-dependent autooscillations of water luminescence triggered by the $1264 \mathrm{~nm}$ radiation. Journal of Physical Chemistry, 115, pp. 7693-7697, 2011.

[12] Binhi, V.N., Magnetobiology: Underlying Physical Problems. Academic Press, San Diego, CA, 2002.

[13] Pollack, G.H \& Clegg, J., Unexpected linkage between unstirred layers, exclusion zones, and v. Phase Transitions in Cell Biology, eds. G.H. Pollack \& W.C. Chin, Springer Science Business Media, Berlin, pp. 143152, 2008.

[14] Del Giudice, E., Spinetti, P.R. \& Tedeschi, A., Water dynamics at the root of metamorphosis in living organisms. Water, 2, pp. 566-586, 2010. 